\title{
THE TREATMENT OF PERIODIC ORBITS BY THE METHODS OF FIXED POINT THEORY
}

\author{
BY F. BROCK FULLER
}

Communicated by S. Smale, April 14, 1966

Let $M$ be a $C^{\infty}$ manifold and let $F$ be a $C^{\infty}$ field of tangent velocity vectors defined over $M$. The question considered here is this: Is it possible to define an "index" for the periodic orbits of the differential equation $d x / d t=F(x)$ which has properties like those of the fixed point index [3]?

It is worthwhile to examine first the special case in which the velocity field $F$ has a surface of section [1] because in this case the periodic orbits can be made to correspond to fixed points. Assume that $M$ is compact and that it is possible to define a $C^{\infty}$ mapping $\theta$ of $M$ onto the circle of real numbers modulo 1 in such a way that $d \theta / d t>0$ on every trajectory of $F$. Let $S$ denote the surface of section $\theta=0$ and let $T$ denote the return map of $S$ onto itself, obtained by following each point of $S$ out along its positive trajectory until its first return to $S$. Define the degree of a periodic orbit to be the number of complete revolutions of $\theta$ as the orbit is traversed. Then to each periodic orbit of degree $d$ corresponds a sequence $x, T(x), \cdots$, $T^{d-1}(x)$ of fixed points of $T^{d}$. These fixed points are distinct if the periodic orbit is simple, i.e. has least period. On the other hand if the periodic orbit has multiplicity $m$, by which we mean that it traverses a simple orbit $m$ times, then exactly $d / m$ of the fixed points are distinct. To the set of all periodic orbits of degree $d$ we can assign the Lefschetz number $\Lambda\left(T^{d}\right)$, or total index of the fixed points of $T^{d}$. Now suppose that $x$ is an isolated fixed point of $T^{d}$. The local behavior of $T^{d}$ at the other fixed points $T(x), \cdots, T^{d-1}(x)$ is isomorphic to that at $x$, in particular all these fixed points are isolated and with the same index $i(x)$. If the corresponding orbit of degree $d$ is simple, its contribution to $\Lambda\left(T^{d}\right)$ is then $d \cdot i(x)$; while if it is of multiplicity $m$, only $d / m$ of the fixed points are distinct, so that its contribution to $\Lambda\left(T^{d}\right)$ is reduced by the factor $1 / \mathrm{m}$.

The total index $\Lambda\left(T^{d}\right)$ assigned to the periodic orbits of degree $d$ can be refined by examining the free homotopy classes in $M$ of the periodic orbits. The following relation can be shown: Two fixed points of $T^{d}$ belong to the same Nielsen class [7] if and only if the periodic orbits of degree $d$ on which they lie belong to the same free homotopy class. Note that the degree is an invariant of the free homotopy class. Thus to each free homotopy class of periodic orbits may be assigned the total index of a Nielsen class. 
What can be expected if we drop the assumption that $F$ has a surface of section, assuming only that $M$ is compact and that $F$ has no singular points? For any periodic orbit can be constructed a transversal at one of its points $x$ and a local return map $T$ mapping a neighborhood of $x$ into the transversal [4]. Suppose first that the periodic orbit is simple. If $x$ is an isolated fixed point of $T$, in which case we say that the periodic orbit is isolated (this definition is different from that of [4]), an index $i(x)$ is defined for $x$ and the natural attempt is to assign $i(x)$ as the "index" of the periodic orbit. Having done this, we are now compelled, given a periodic orbit of multiplicity $m$, to assign to it the index $i(x) / m$, where $i(x)$ is the index of $x$ as a fixed point of $T^{m}$. At this point we might be led to expect the following: To each free homotopy class of periodic orbits can be assigned a rational number as its total index; the total index remains invariant under deformations of the velocity field $F$ which do not introduce singular points.

The above expectation is not quite true. Suppose that the velocity field $F$ has a surface of section. It may happen that as $F$ is deformed toward a field $G$ which has vectors tangent to some of the surfaces $\theta=$ constant, it takes a longer and longer time for some of the points of $S$ to return to $S$. Correspondingly some of the periodic orbits have longer and longer periods and for $G$ they disappear altogether, thereby changing the total index. This possibility indicates that the periods should be included in the topology of the space containing the periodic orbits. Accordingly we regard the periodic orbits as being located, not in $M$, but in $M \times(0, \infty)$, where we assign the period (not necessarily least) to the second component.

The above discussion is intended to elucidate the statement of the following theorem, which is proved in [2]. The statement follows a course parallel to that of the fixed point theorems of [3].

Theorem. Let $M$ be a $C^{\infty}$ manifold, let $F$ be a $C^{\infty}$ field of tangent velocity vectors defined over $M$ and let $\omega$ be a relatively compact open set in $M \times(0, \infty)$ whose boundary does not meet any periodic orbits or points $(x, t)$ where $x$ is a singular point of $F$. Then a rational number $i(\omega)$ can be defined, the index of $\omega$, with the following properties:

(1) If $\omega$ contains only one periodic orbit, then $i(\omega)$ is equal to the index defined above, using the return map on a transversal.

(2) If $\omega_{1}$ and $\omega_{2}$ are disjoint open sets for which the index is defined then $i\left(\omega_{1} \cup \omega_{2}\right)=i\left(\omega_{1}\right)+i\left(\omega_{2}\right)$.

(3) If $F$ or $\omega$ are continuously deformed in such a way that $i(\omega)$ remains defined, then $i(\omega)$ does not change. 
REMARK. Let $\gamma$ be a free homotopy class of closed curves in $M \times(0, \infty)$. The theorem remains true if the phrase "periodic orbit" is replaced by "periodic orbit of class $\gamma$ " and $i(\omega)$ is replaced by an index $i(\omega, \gamma)$ depending on $\gamma$. Thus there is no interaction between different free homotopy classes of periodic orbits.

Application. The theorem can be applied to yield the theorem of Seifert [5], [6] on periodic orbits in the 3-sphere $S^{3}$, at least in the case of $C^{\infty}$ velocity fields. Let $F$ be a field of unit tangents to the Clifford circles in $S^{3}$. Then $S^{3}$ is filled with periodic orbits of period $2 \pi$, together with their multiples. Locating the orbits in $S^{3} \times(0, \infty)$, the set $\omega=S^{3} \times(\pi, 3 \pi)$ is a relatively compact open set with no periodic orbits meeting its boundary. Hence $i(\omega)$ is defined and remains invariant under deformations of $F$, so long as no singular points or the periods $\pi$ or $3 \pi$ appear. To show that such deformed fields must have at least one periodic orbit it suffices to show that $i(\omega) \neq 0$. But a small deformation of $F$ results in two isolated periodic orbits in $\omega$, each of index +1 . Hence $i(\omega)=2$.

\section{REFERENCES}

1. G. D. Birkhoff, Dynamical systems, Amer. Math. Soc. Colloq. Publ., Vol 9' Amer. Math. Soc., Providence, R. I., 1927.

2. F. B. Fuller, An index of fixed point type for periodic orbits, (to appear).

3. J. Leray, La theorie des points fixes et ses applications en analyse, Proc. Internat. Congr. Mathematicians, 1950, vol. II, pp. 202-208.

4. L. Markus, The behavior of the solutions of a differential equation near a periodic solution, Ann. of Math. 72 (1960), 245-266.

5. G. Reeb, Sur un théorème de Siefert sur les trajectories fermées de certains champs de vecteurs, Internat. Sympos. Nonlinear Differential Equations and Nonlinear Mechanics, New York, 1963.

6. H. Seifert, Closed integral curves in 3-space and isotopic two-dimensional deformations, Proc. Amer. Math. Soc. 1 (1950), 287-302.

7. F. Wecken, Fixpunktklassen, Math. Ann. 117 (1941), 659-671; 118 (1943), 216-234, 544-577.

California Institute of Technology 